\title{
A micro-mechanical compaction model for granular mix of soft and rigid par- ticles
}

\author{
Manuel Cárdenas-Barrantes ${ }^{1, \star}$, David Cantor $^{2}$, Jonathan Barés ${ }^{1}$, Mathieu Renouf ${ }^{1}$, and Emilien Azéma ${ }^{1,3}$ \\ ${ }^{1}$ LMGC, Université de Montpellier, CNRS, 163 rue Auguste Broussonnet, Montpellier, France \\ ${ }^{2}$ Department of Civil, Geological and Mining Engineering, Polytechnique Montréal, 2500 Chemin de Polytechnique, Québec, \\ Canada \\ ${ }^{3}$ Institut Universitaire de France (IUF), Paris, France
}

\begin{abstract}
We use bi-dimensional non-smooth contact dynamics simulations to analyze the isotropic compaction of mixtures composed of rigid and deformable incompressible particles. Deformable particles are modeled using the finite-element method and following a hyper-elastic neo-Hookean constitutive law. The evolution of the packing fraction, bulk modulus and particle connectivity, beyond the jamming point, are characterized as a function of the applied stresses for different proportion of rigid/soft particles and two values of friction coefficient. Based on the granular stress tensor, a micro-mechanical expression for the evolution of the packing fraction and the bulk modulus are proposed. This expression is based on the evolution of the particle connectivity together with the bulk behaviour of a single representative deformable particle. A constitutive compaction equation is then introduced, set by well-defined physical quantities, given a direct prediction of the maximum packing fraction $\phi_{\max }$ as a function of the proportion of rigid/soft particles.
\end{abstract}

\section{Introduction}

The mixture of particles with different elastic properties is extensively observed in nature and also in a great number of applications. In nature, the interactions of these kind of assemblies have a great importance in, to name a few, foams [1, 2], organic cells [3] and clayey materials [4]. It has also a great importance in engineering applications, like seismic protection and acoustic isolations [5, 6], damping control [7], metal compaction and pharmaceutical procedures [8]. Nonetheless, the analysis of the behavior of these mixtures is very complex given the sharp difference in the elastic properties of their constitutive elements and the different interactions within the system.

For systems composed entirely of deformable particles there is a large number of equations that describe well their compaction behavior [9-12], but mostly reduced to different variations of the equation proposed by Heckel [13], which assumes a proportionality between the porosity and the packing fraction increment over the stress increment. Systems composed of mixtures of rigid and deformable particles has been less studied, but it is worth highlighting the work of Platzer et al. [14] among others [15, 16]. They deduced an equation from the assumption that the empty space is filled as a first-order differential equation of the applied pressure. The authors mentioned that their model provides fair predictions for low pressures and a mixture ratio below $50 \%$. Is is only recently that a micromechanical compaction model for assemblies of circular

\footnotetext{
^e-mail: manuel-antonio.cardenas-barrantes@umontpellier.fr
}

A video is available at https://doi.org/10.48448/cevr-fq53 deformable particles, based on the contact network evolution and the scaling of the elastic properties of a single particle, has been proposed [17].

Here, we analyze the compaction behavior of mixtures of rigid and deformable particles by using a coupled discrete element and finite element method in the frame of the non-smooth contact dynamics (NSCD) approach. We study the effect of the proportion of rigid-deformable particles in the mixture and the friction on the compaction evolution and the elastic properties beyond the jamming point. We introduce an analytical model for the compaction in terms of the evolution of the particle connectivity, the applied pressure, the packing fraction, and the mixture ratio. This model is an extension to binary mixtures of our previous work on assemblies of only deformable particles [17].

The outline of the paper is as follows. In Section 2, we describe the numerical method and procedure followed during the compaction. The compaction curve as well as the bulk modulus are also presented. In Section 3, a micromechanical model of compaction is introduced and validated. Finally, conclusions are presented in Section 4.

\section{Procedure and Results}

In the frame of non-smooth contact dynamics (NSCD) [18], in which the Finite Element and the Contact dynamics methods are coupled, we simulate packings of mixtures of rigid and deformable circular particles under external isotropic compression. It considers a contact law with non-penetrability and dry friction between particles. 

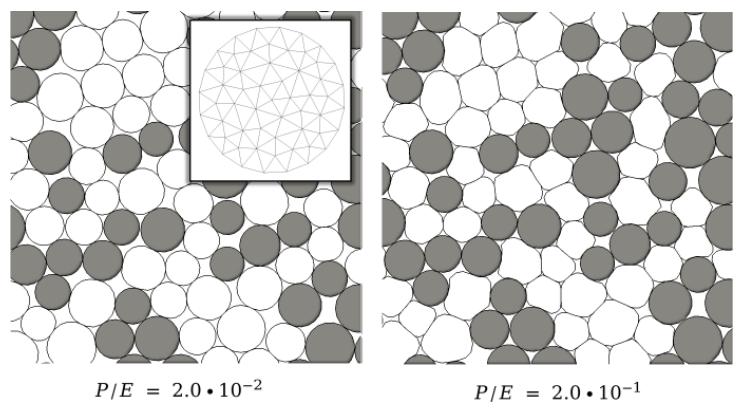

Figure 1. Views for a mixture ratio $\kappa=0.5$ and different reduced pressure $P / E$ with $\mu=0.2$. The inset shows the finite element mesh used. The rigid particles are colored in grey.

The deformable particles are modeled following the neoHookean incompressible material law. The simulations are implemented on the open-source simulation platform LMGC90 [19-21].

First, $N_{p}=1500$ rigid disks, with a small size polydispersity, are randomly placed into a square box of initial length $L_{0}$ and gently compressed to reach the jammed state. Second, a volume $\kappa V_{p}$ is homogeneously replaced by deformable disks meshed with 92 triangular similarsize elements, with $\kappa$ the mixture ratio varying in the set $[0.2,0.5,0.8,1.0]$ (from $\kappa=0.2$, a packing composed of $20 \%$ of deformable particles, to 1 , a packing of only deformable particles). We use a constant Poisson's ratio equals to 0.495 and a Young modulus $E$ (with $E \rightarrow \infty$ for rigid particles) and two values of friction, $\mu=0.0$ and $\mu=0.2$. The friction with the walls and the gravity are set to 0 to avoid external force gradients in the sample.

Finally, starting from the jammed state, the packings are isotropically compressed by gradually, and quasistatically, applying a stress $P$ on the boundaries in a set of loading steps. For a given pressure $P$, a stable state is reached once the variations of the packing fraction $\phi$, defined as the total volume of the particles $V_{p}$ divided by the volume of the containing box $V\left(\phi=V_{p} / V\right)$, remains below $0.01 \%$. In the Tab. 2, we summarize the initial state of the samples simulated (packing fraction $\phi_{0}$ and coordination number $Z_{0}$ ). To be sure that the system is always in the quasi-static regime, we also control the velocity of the walls based on the inertial number, $I$ [22]. We impose that $I<<10^{-4}$ for each step on the simulation. Figure 1 shows a packing with $\kappa=0.5$ and increasing values of reduced pressure $P / E$ at $\mu=0.2$.

Figure 2 shows the evolution of $\phi$ as a function of $P / E$ along with the compaction model proposed by Platzer et al., given by $P=-P_{0}(\kappa) \ln \left[\left(\phi_{\max }-\phi\right) /\left(\left(\phi_{\max }-\phi_{0}\right)\right]\right.$, with $\phi_{0}$ the packing fraction at the jammed state, $\phi_{\max }$ the maximum value reached by $\phi$ and $P_{0}(\kappa)$ a characteristic pressure depending, a priori, on the proportion [14]. As seen in previous studies, $\phi$ first increases with $P / E$ from a well-known value $\phi_{0}$ and then tends asymptotically to a maximum value $\phi_{\max }$. Both $\phi_{0}$ and $\phi_{\max }$ slightly decrease as the friction increases, which is explained by the rearrangements of the grains within the assembly, being more frequent in the frictionless case [23, 24]. In addition,

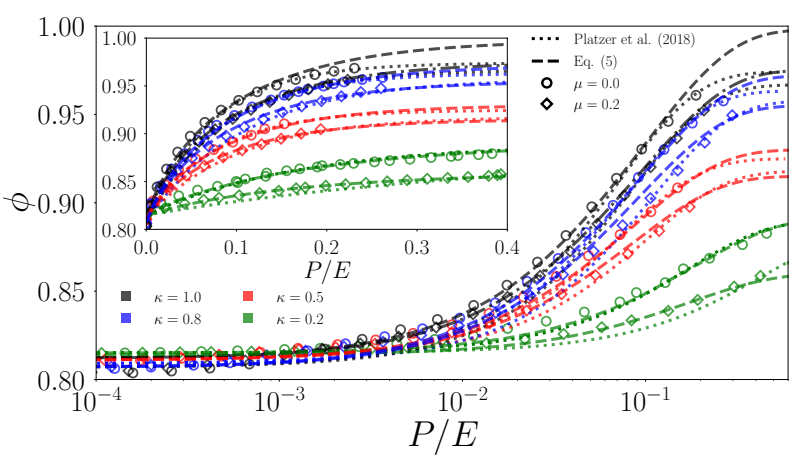

Figure 2. Packing fraction $\phi$ as a function of $P / E$ for mixtures of $\kappa \in[0.2, \ldots, 1]$ and $\mu \in\{0,0.2\}$. The predictions given by the micro-mechanical equation (Eq. (5)) and the ones given by the Platzer et al. model are also shown.

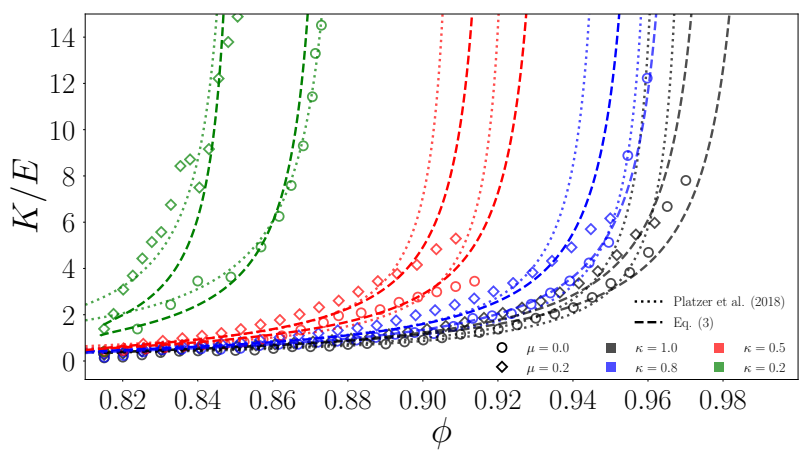

Figure 3. Bulk modulus $K$ over $E$ as a function of the packing fraction $\phi$ for different values of $\kappa$ and for $\mu=0$ and 0.2 . We also present the predictions given by Eq. (3) and the bulk modulus computed from the Platzer et al. model.

Table 1. Values of $\phi$ and $Z$ at the jammed state for the different samples of mixtures simulated.

\begin{tabular}{lllll}
\hline$\phi_{0}$ & $\kappa=0.2$ & $\kappa=0.5$ & $\kappa=0.8$ & $\kappa=1$ \\
\hline$\mu_{s}=0.0$ & 0.815 & 0.811 & 0.807 & 0.807 \\
$\mu_{s}=0.2$ & 0.814 & 0.811 & 0.807 & 0.805 \\
\hline \hline$Z_{0}$ & $\kappa=0.2$ & $\kappa=0.5$ & $\kappa=0.8$ & $\kappa=1$ \\
\hline$\mu_{s}=0.0$ & 3.4 & 3.6 & 3.5 & 3.4 \\
$\mu_{s}=0.2$ & 3.3 & 3.7 & 3.5 & 3.5
\end{tabular}

from the $\phi-P$ curve we computed the bulk modulus as $K(\phi)=\frac{d P}{d \phi} \cdot \frac{d \phi}{d \varepsilon_{v}}$, with $\varepsilon_{v}=-\ln \left(\phi_{0} / \phi\right)$, the macroscopic cumulative volumetric strain. The bulk modulus as a function of $\phi$, along with the prediction given by the model of Platzer et al., is plotted in Fig. 3. The modulus $K$ increases with $\phi$ and diverges as it approaches to $\phi_{\max }$. This comes from the fact that there is no more void space and the assembly of grains starts to behave as a continuous and rigid material.

\section{Theoretical approach}

Let us consider the micro-mechanical expression of the granular stress tensor as $\sigma_{i j}=\frac{1}{V} \sum_{c \in V} f_{i}^{c} \ell_{j}^{c}=\frac{N_{c}}{V}\left\langle f_{i}^{c} \ell_{j}^{c}\right\rangle_{c}$, 


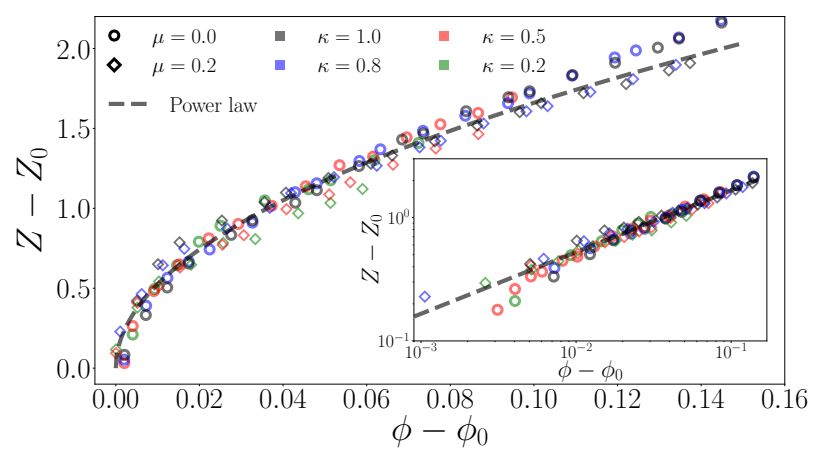

Figure 4. The reduced coordination number $Z-Z_{0}$ as a function of the reduced solid fraction $\phi-\phi_{0}$ for all the values of $\kappa$ and $\mu$ simulated. The dashed line is the power-law relation $Z-Z_{0}=$ $\xi\left(\phi-\phi_{0}\right)^{\alpha}$.

where the sum runs over all the contacts enclosed by the volume $V$, with $f_{i}^{c}$ the $i^{t h}$ component of the contact force at the contact $c$ and $\ell_{j}^{c}$ the $j^{\text {th }}$ component of the vector joining the centers of particles interacting. $N_{c}$ is the total number of contacts in the volume $V$, and the notation $\langle\ldots\rangle_{c}$ stands for the average over the contacts. From the stress tensor, the mean stress is given by $P=\left(\sigma_{1}+\sigma_{2}\right) / 2$, with $\sigma_{1}$ and $\sigma_{2}$ the principal stress values.

Assuming a small particle size dispersion around the diameter $\langle d\rangle$ and $Z=2 N_{c} / N_{p}$, the coordination number, a microstructural equation for the compressive stress can be deduced [25]:

$$
P=\frac{Z \phi}{\pi} \sigma_{\ell},
$$

with $\sigma_{\ell}=\left\langle f^{c} \cdot \ell^{c}\right\rangle_{c} /\langle d\rangle^{2}$, a measure of the inter-particle stresses.

Let us now reduce the equation (1) to an explicit relation between $P$ and $\phi$. At the jammed state (i.e. for a packing fraction $\phi_{0}$ ), the packing is also characterized by a typical value $Z_{0}$ of the coordination number (Tab. 2). Below such value the motion of the particles is made possible without implying particle deformations. Many studies show that $Z_{0}$ depends on parameters related to the shape of the particles, the friction and the packing preparation [26-28].

Above the jammed state, it has been systematically reported that $Z$ increases following a power-law as $Z-Z_{0}=$ $\xi\left(\phi-\phi_{0}\right)^{\alpha}$, with $\alpha \sim 0.5$ and $\xi$ a structural parameter fully defined as $P / E \rightarrow \infty$, with both $\phi$ and $Z$ reaching a maximum value $\phi_{\max }$ and $Z_{\max }$, respectively. This relation is observed both numerically and experimentally for many type of deformable granular systems, such as foams, emulsions and rubber-like particles [3, 24, 29, 30]. We found the same relation in our simulations, with $\xi \sim 5.1$, independently of the mixture ratio and friction coefficient (Fig. 4). Thus, our results extend the validity of such relation to binary mixtures.

At the same time, the inter-particle stress, $\sigma_{\ell}$, could be related to the packing fraction through the bulk properties of an elementary system composed of a single elastic particle under the same stress and boundary conditions as the

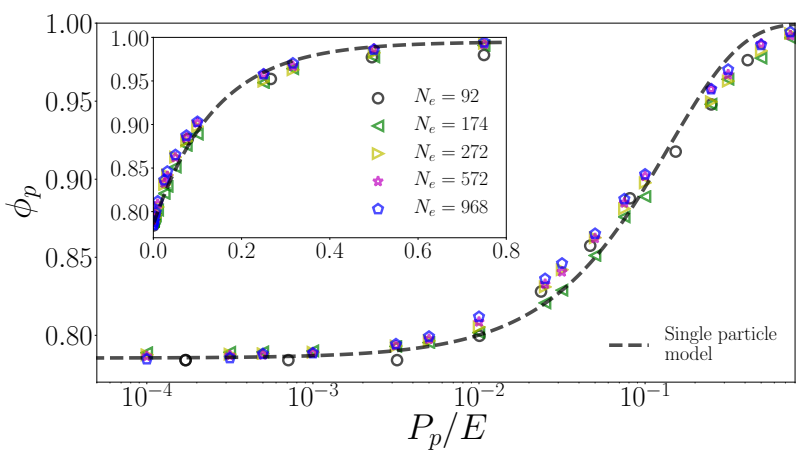

Figure 5. The packing fraction as a function of the scaled pressure applied on the particle for different mesh resolutions, $N_{e}$.

multi-particle assembly. In Fig. 5 we present the evolution of the packing fraction computed on the single particle system, $\phi_{p}$, as a function of the applied pressure $P_{p}$. We observe that the single particle compression curve $\phi_{p}-P_{p}$ is similar to the multi-particle compaction curve $\phi-P$ (Fig. 2 ). The compaction behavior of such elementary system is well described with the following logarithmic function (gray dashed line in Fig. 5):

$$
P_{p} / E=-b \ln \left(\frac{\phi_{p, \max }-\phi_{p}}{\phi_{p, \max }-\phi_{p, 0}}\right),
$$

with $\phi_{p, \max }$ the maximum packing fraction obtained, $\phi_{p, 0}=\pi / 4$ and $b$ a constant of proportionality called the yield stress and found to be $b \simeq 0.14$. Equation (2) results from the analogy of a single particle system to the collapse of a cavity within an elastic medium under isotropic compression [9]. Then, the bulk modulus of the single particle assembly is given by: $K_{p}\left(\phi_{p}\right)=\left(d P_{p} / d \phi_{p}\right) \cdot\left(d \phi_{p} / d \varepsilon_{v, p}\right)$, with $\varepsilon_{v, p}=-\ln \left(\phi_{p, 0} / \phi_{p}\right)$.

Remarkably, as shown in Fig. 3, the Bulk modulus $K$ of the multi-particle assembly scales with the Bulk modulus $K_{p}$ of the single particle assembly when they are compared for equivalent packing fraction:

$$
K \equiv \frac{Z \phi}{2 \pi} K_{p}+O
$$

with $O$ negligible high order terms depending on $\phi$. Equation 3 allows us to described the micro-mechanical origin of the Bulk modulus of an assembly of rigid-deformable particles in terms of particle connectivity, packing fraction, and the Bulk property of an elementary system. From Eq. (1) we get:

$$
P \simeq \frac{Z \phi}{2 \pi} P_{p} .
$$

Equation (3) and (4) reveal that the elastic and compaction properties of a binary mixture scale from the behavior of a single particle under similar stress conditions. This finding is in agreement with the general idea of describing the macroscopic properties of a granular packing from a single representative element $[31,32]$.

Finally, including the $Z-\phi$ relation into Eq. (4), together with Eq. (2) at equivalent packing fraction, we get 
the following compaction equation:

$$
\frac{P(\phi, \kappa)}{E} \simeq-\frac{b \phi}{2 \pi}\left\{Z_{0}+\xi\left(\phi-\phi_{0}\right)^{\alpha}\right\} \ln \left(\frac{\phi_{\max }(\kappa)-\phi}{\phi_{\max }(\kappa)-\phi_{0}}\right) .
$$

Eq. 5 is based on a number of structural parameters that are determined from the initial state $\left(Z_{0}\right.$ and $\left.\phi_{0}\right)$, the behavior of a single representative particle (b), or the mapping between the packing fraction and coordination curve ( $\xi$ and $\alpha$ ). $\phi_{\max }$ remains the only parameter that is deduced by correctly fitting Eq. 5 to the macroscopic data as shown in Fig. 2, both as a function of $\kappa$ and for the two values of $\mu$.

\section{Conclusions}

Starting from the jammed state to very high densities, we analyze the compression behavior of bidimensional granular assemblies composed of mixtures of rigid and incompressible deformable particles through non-smooth contact dynamics simulations and following a hyper-elastic neo-Hookean constitutive law. The proportion of deformable particles was varied from assemblies composed of $20 \%$ of deformable grains up to assemblies fully composed of deformable grains, for different values of friction.

In this work we introduce a new compaction model, with well-defined micro-mechanical bases, that describes the compaction of the rigid-soft particle composite. The model shows that the essential behavior of the compaction of this mixture (which also covers completely deformable systems) is captured from the characterization of a single particle, together with the evolution of particle connectivity. The natural path of this work is the analysis of more real systems, such as 3D systems, composite assemblies of particles with more realistic geometries, all under different compaction conditions.

\section{References}

[1] J. Mauer, S. Mendez, L. Lanotte, F. Nicoud, M. Abkarian, G. Gompper, D.A. Fedosov, Phys. Rev. Lett. 121, 118103 (2018)

[2] D. Bi, X. Yang, M.C. Marchetti, M.L. Manning, Phys. Rev. X 6, 021011 (2016)

[3] G. Katgert, M. van Hecke, Europhys. Lett. 92, 34002 (2010)

[4] M.O.B. Rúa, P. Bustamante-Baena, Boletín de la Sociedad Española de Cerámica y Vidrio (2019)

[5] K. Senetakis, A. Anastasiadis, K. Pitilakis, Soil Dyn. Earthq. Eng. 33, 38 (2012)

[6] A. Tsiavos, N.A. Alexander, A. Diambra, E. Ibraim, P.J. Vardanega, A. Gonzalez-Buelga, A. Sextos, Soil Dyn. Earthq. Eng. 125, 105731 (2019)
[7] B. Indraratna, Y. Qi, T. Ngo, C. Rujikiatkamjorn, F. Neville, Bessa-Ferreira, A. Shahkolahi, Geosc. 9, 30 (2019)

[8] W. Zhang, K. Liu, J. Zhou, R. Chen, N. Zhang, G. Lian, J. Phys. Soc. Japan 89, 044602 (2020)

[9] M.M. Carroll, K.T. Kim, Powder Metall. 27, 153 (1984)

[10] L. Parilak, E. Dudrova, R. Bidulsky, M. Kabatova, Powder Technol. 322, 447 (2017)

[11] J. Secondi, Powder Metall. 45, 213 (2002)

[12] Y.X. Zhang, X.Z. An, Y.L. Zhang, Appl. Phys. A 118, 1015 (2014)

[13] R.W. Heckel, Trans. Metal. Soc. Aime. 221, 671 (1961)

[14] A. Platzer, S. Rouhanifar, P. Richard, B. Cazacliu, E. Ibraim, Granul. Matter 20, 81 (2018)

[15] I. Popescu, R. Vidu, Mater. Mech. 16 (2018)

[16] D. Wang, X. An, P. Han, H. Fu, X. Yang, Q. Zou, Math. Probl. Eng 2020, 5468076 (2020)

[17] D. Cantor, M. Cárdenas-Barrantes, I. Preechawuttipong, M. Renouf, E. Azéma, Phys. Rev. Lett. 124, 208003 (2020)

[18] M. Jean, Comput. Methods Appl. Mech. Eng. 177, 235 (1999)

[19] git-en.lmgc.univ-montp2 .fr/lmgc90 /lmgc90user/wikis/home

[20] F. Dubois, V. Acary, M. Jean, C. R. Mecanique 346, 247 (2018)

[21] M. Renouf, F. Dubois, P. Alart, J. Comput. Appl. Math. 168, 375 (2004)

[22] B. Andreotti, Y. Forterre, O. Pouliquen, Granular media: between fluid and solid (Cambridge University press, 2013)

[23] T.L. Vu, S. Nezamabadi, S. Mora, Soft Matter 16, 679 (2020)

[24] T.S. Majmudar, M. Sperl, S. Luding, R.P. Behringer, Phys. Rev. Lett. 98, 058001 (2007)

[25] I. Agnolin, J.N. Roux, Phys. Rev. E 76, 061304 (2007)

[26] M. van Hecke, Condens. Matter Phys. 22, 033101 (2009)

[27] L.E. Silbert, D. Ertaş, G.S. Grest, T.C. Halsey, D. Levine, Phys. Rev. E 65, 031304 (2002)

[28] M.H. Khalili, J.N. Roux, J.M. Pereira, S. Brisard, M. Bornert, Phys. Rev. E 95, 032907 (2017)

[29] T.L. Vu, J. Barés, S. Mora, S. Nezamabadi, Phys. Rev. E 99, 062903 (2019)

[30] D.J. Durian, Phys. Rev. Lett. 75, 4780 (1995)

[31] B.P. Tighe, T.J.H. Vlugt, J. Stat. Mech. Theory Exp. 2011, P04002 (2011)

[32] M. Cárdenas-Barrantes, J.D. Muñoz, W.F. Oquendo, Granul. Matter 20, 1 (2018) 